\title{
Complex auditory impairment with peduncular hallucinosis due to pontine haemorrhage - a case report
}

\author{
Anca Negrila ${ }^{1}$, Elena Oana Terecoasa ${ }^{1,2}$, Razvan Alexandru Raduu, ${ }^{2,3}$ Bogdan Casaru', \\ Andreea Nicoleta Marinescu ${ }^{2,4}$, Cristina Tiu ${ }^{1,2}$ \\ ${ }^{1}$ Stroke Unit, Department of Neurology, University Emergency Hospital, Bucharest, Romania \\ 2 "Carol Davila" University of Medicine and Pharmacy, Bucharest, Romania \\ ${ }^{3}$ Department of Interventional Radiology, University Emergency Hospital, Bucharest, Romania \\ ${ }^{4}$ Department of Radiology and Medical Imaging, University Emergency Hospital, Bucharest, Romania
}

\begin{abstract}
Peduncular hallucinosis is a rare syndrome that develops with upper brainstem and diencephalon lesions. Patients complain of vivid hallucinations that usually occur in hypnagogic states but maintain critical appraisal. Pathological disruption of the reticular activating systems partially explains this clinical phenomenon. We report the case of a 47-year-old man presenting with peduncular hallucinosis associated with central auditory disturbance consisting of bilateral hypoacusis and left-sided paradoxical hyperacusis, secondary to a pontine haemorrhage.
\end{abstract}

Keywords: peduncular hallucinosis, hypoacusis, hyperacusis, pontine haemorrhage

\section{INTRODUCTION}

Peduncular hallucinosis is a rare syndrome that was first described by Lhermitte in 1922 in a patient with oculomotor palsy who presented with vivid, dream-like visual hallucinations associated with a hypnagogic state. The cause was believed to be an infarction of the cerebral peduncles that disrupted the normal transition between sleep and wakefulness (1). Cases of peduncular hallucinosis have thenceforth been reported in both thalamic and upper pons lesions (2).

The classic syndrome consisted of visual hallucinations. However, auditory and tactile hallucinations have since been reported in association, suggesting the involvement of a more complex network (3). Several more or less common brainstem syndromes manifesting with hallucinations have been described in literature. The diagnostic approach to posterior circulation stroke mostly relies now on modern imaging techniques and careful clinical examination is sometimes disregarded. Beyond hy- peracute management, many symptoms and signs are frequently overlooked and subtler disorders of processing functions can be misdiagnosed or even eluded. Stroke involving territories above the medullary - pontine junction can be associated with manifold auditory dysfunctions, including sensory deficits manifesting either as hypoacusis with variable degrees of severity or, on the contrary at times, as a lowered threshold for sound perception (4).

We report the case of a 47-year-old man with an intracerebral haemorrhage due to a pontine cavernoma presenting with peduncular hallucinosis and bilateral hypoacusis associated with paradoxical hyperacusis. This case report was prepared according to CARE guidelines (5).

Written informed consent was obtained from the pacient for the publication of this case report in accordance with the Declaration of Helsinki and all research was conducted following legal and ethical requirements of the University Emergency Hospital Bucharest. 


\section{CASE REPORT}

A 47-year-old Romany male patient with a prior diagnosis of ulcerative colitis was admitted to our department for sudden onset of anarthria and left-sided hemiplegia. The patient had no previous history of arterial hypertension or illicit drug use.

Initial clinical examination revealed left hemiplegia, decreased pinprick sensation on his left side, right-sided ataxia, right peripheral facial palsy with anarthria and left-sided gaze deviation with partial vertical gaze palsy, suggestive of a pontine Foville syndrome. Blood pressure was 160/90 $\mathrm{mmHg}$ at admission but normalized in approximately 48 hours and remained in normal range for the rest of the hospitalization without any medical intervention. Laboratory workup was unremarkable. The non-contrast cerebral computed tomography (NCCT) scan performed at admission revealed a right sided tegmental pontine haemorrhage. Brain magnetic resonance imaging (MRI) (Figure 1) was performed two weeks later and identified a vascular malformation suggestive of a cavernous malformation, which was further confirmed by the absence of arterial feeders and venous drainage on digital subtraction angiography.

While hospitalized, the patient described an unusual intolerance to regular environmental sounds, which were oftentimes perceived by the patient as very loud, disturbing noises. As the patient's speech was severely impaired due to dysarthria, these complaints were initially mistakenly interpreted as tinnitus and vertigo. Later on, it became evident that the patient complained of multiple auditory hallucinations, both verbal and nonverbal (of bruitlike quality). The hallucinations appeared usually in the evening and were associated with eye closure before falling asleep. He maintained critical thinking concerning these phenomena. Bilateral hypoa- cusis, more pronounced on the right side, was also noted.

The patient had no prior history of psychiatric illness. Ear, nose and throat examination showed no apparent abnormalities. The otoscopic examination did not reveal any lesions suggestive of conductive hearing impairment.

The hallucinations and hearing abnormalities subsided to some degree throughout the hospital stay and were still present at 3 months follow-up.

\section{DISCUSSION}

The patient described herein represents a rare case of a pontine haemorrhage presenting with hallucinosis and central auditory manifestations consisting of hypoacusis associated with paradoxical hyperacusis.

Peduncular hallucinosis, also known as Lhermitte's syndrome, is characterized by hallucinations that usually appear in the context of brainstem lesions involving the reticular formation. The hallucinations are most often visual, but auditory hallucinations have also been described, and usually appear later in the day, with eye closure (6). An important mention while describing this syndrome is defining the term hallucinosis. While hallucinations are an integrated part of this concept, hallucinosis refers distinctively to the manifestation of hallucinations in a person with a normal mental status, without disorientation, confusion or any type of psychosis (7). The most probable explanation for these symptoms is an imbalance in neurotransmitters' activity. Lesions of the dorsal raphe nucleus cause loss of inhibitory serotonergic activity, which results in a predominance of acetylcholine activity. Thus, acetylcholine released by the pedunculo-pontine and latero-dorsal tegmental nuclei mediates cortical activation of temporal and occipital regions,

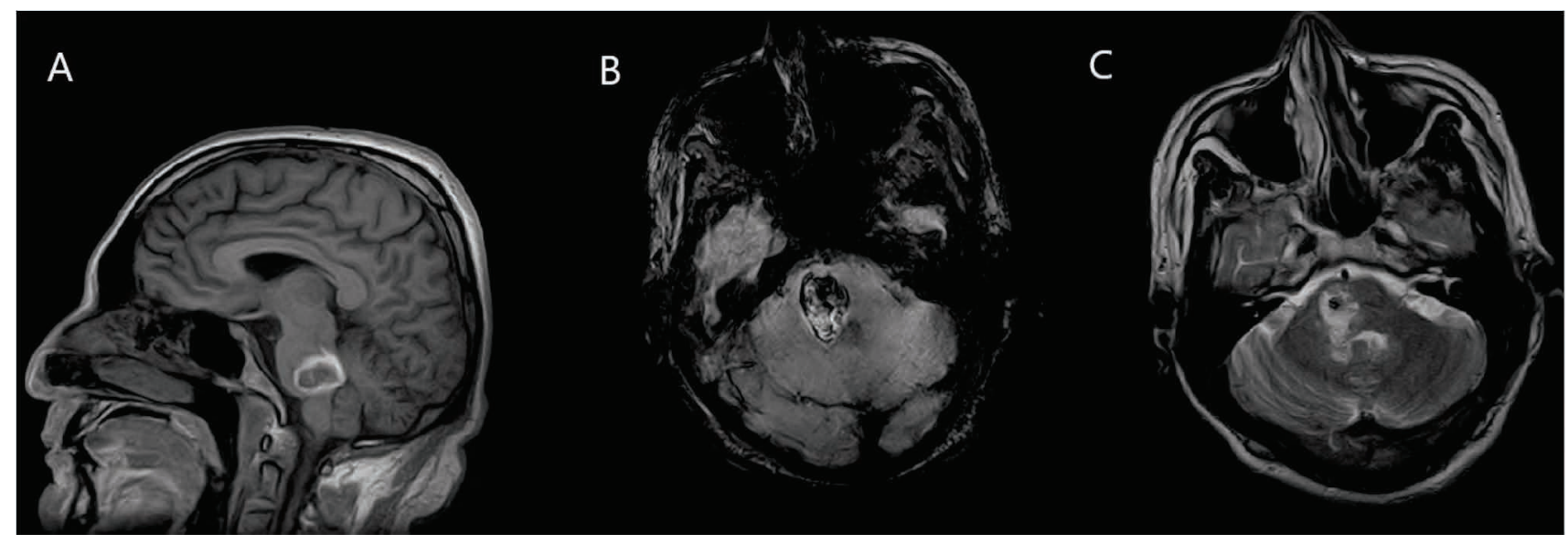

FIGURE 1. A: T1 sequence showing pontine hypointense lesion with hyperintense outer rim, suggestive of a subacute haemorrhage. B: Susceptibility weighted imaging hypointense "blooming" appearance around and within the lesion, suggestive for a cavernoma. C: T2 sequence showing hyperintense lesion with hypointense centre, suggestive for a subacute haemorrhage with surrounding oedema 
causing perceptual disinhibition (Figure 2-A) (3). Peduncular hallucinosis is most frequently caused by vascular lesions of the brainstem [8], but as cases are rare, most data on this topic is based on case reports and small case series where other causes such as infections, tumours and multiple sclerosis have been mentioned (9-12). Most patients with brainstem hallucinations have lesions that involve the auditory structures, located in the pontine tegmentum. These lesions involve sensory structures and are supposed to be responsible for cortical deafferentation and subsequent activation of cortical auditory structures (13).

Auditory hallucinations can be a hallmark of either cortical or subcortical brain damage. The underlying lesions responsible for their occurrence can be located at any level of the auditory pathway. While the most common type of paracusis in brainstem stroke is tinnitus, more complex types of hallucinations, as was the case of our patient, can be observed $(14,15)$. Auditory hallucinations can be classified as being of verbal and non-verbal quality. In some cases it can be difficult to correctly classify the hearing impairment in the acute phase of the illness due to brainstem related accompanying signs (most often severe dysarthria), hyperacusis and auditive hallucinations being mistakenly interpreted as tinnitus (1). Verbal hallucinations have been classically associated with temporal lobe lesions, but brainstem stroke can also lead to such manifestations.

Multiple hallucinations have been reported in brain lesions that did not involve the classical sensory pathways, suggesting an indirect connexion to remote brain centres. A study on this issue used lesion network mapping to analyse the maps of rest- ing functional imaging in normal subjects and subjects with brain lesions and new-onset hallucinations, with the aim of identifying the common anatomical basis. It was thus observed that for most of the patients with auditory hallucinations lesions were connected to the dentate nucleus of the cerebellum. Beside the role of the dentate nucleus, a standard system for both visual and auditory phenomena which involved the cerebellar vermis has also been described. It remains to be seen if disruption of cortical - cerebellar circuits is also responsible for auditory hallucinations in patients with pontine lesions (Figure 2-B) (16).

Auditory dysfunction is a rare manifestation of focal brainstem haemorrhage and limited data regarding the prevalence of hearing abnormalities associated with brainstem lesions is available $(17,18)$. Numerous fibres of the acoustic pathway pass through the brainstem, but as there is a bilateral representation above the medullary-pontine junction, brainstem stroke often presents with vestibular symptoms and only rarely with hearing disturbances. Most neurons of the auditory pathway above the cochlear nuclei have a binaural quality meant to accomplish the complex coding needed for the space localisation of sounds. This type of space-sensitive perception can be achieved by two prompting inputs described as: interaural time difference and interaural level difference. Moreover, in line with this function, the nuclei along the auditory pathway receive prominently excitatory inputs from the contralateral ear (19).

The ventral cochlear nucleus is extensively connected to both inferior colliculi and olivary complex and the contralateral cochlear nuclei (15). Connections from the ventral cochlear nucleus travel to the

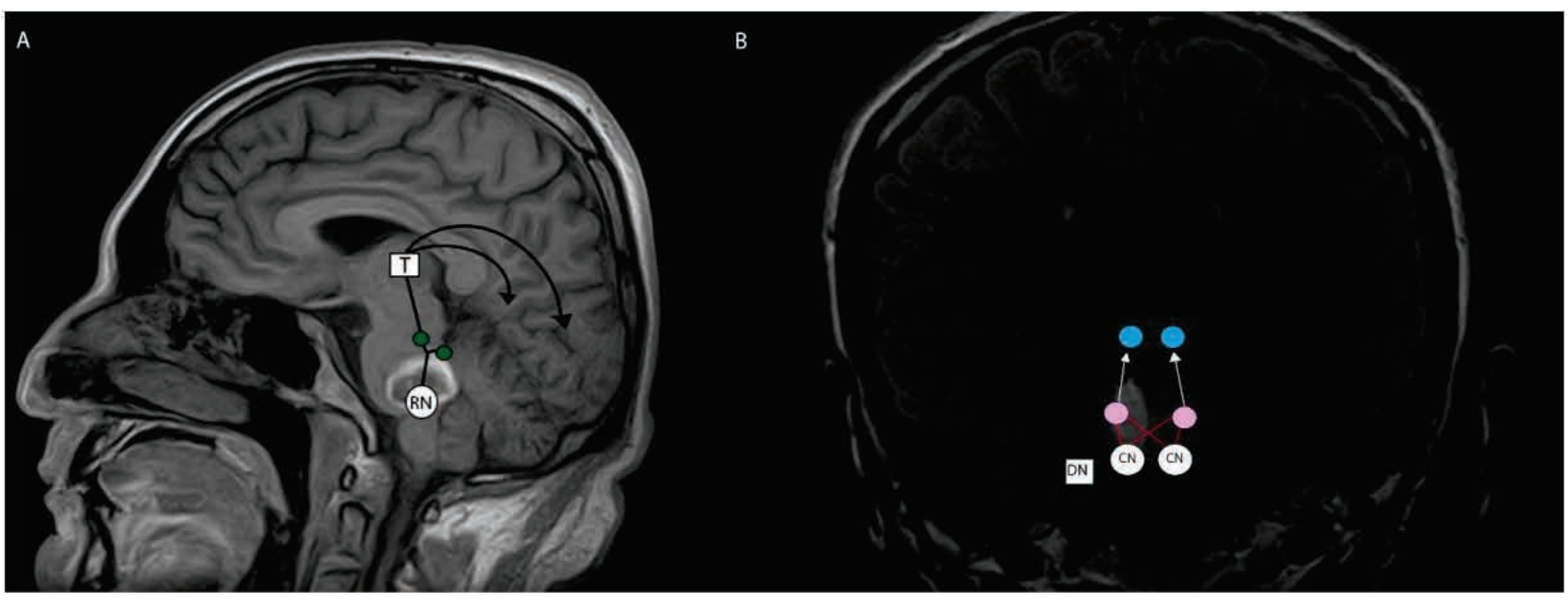

FIGURE 2. A: T1 sequence, sagittal section, showing the main pathway causing neurotransmitter imbalance in peduncular hallucinosis ( $R N$ - dorsal raphe nucleus; $\mathrm{T}$ - thalamus; green dots - pedunculopontine and laterodorsal tegmental nuclei). B: T2 FLAIR sequence, coronal section, showing main pathways involved in central auditory dysfunction caused by brainstem lesions (white dots - $\mathrm{CN}$ - ventral cohlear nuclei; pink dots - superior olivary complex; blue circle - inferior colliculi; DN - dentate nucleus) 
trapezoid body, a structure which encompasses numerous auditory nuclei that integrate cochlear information. Some of these fibres synapse in the contralateral superior olivary complex, while others only pass through the trapezoid body to join the lateral lemniscus to the inferior colliculi (4). A subdivision of the trapezoid body, the medial nucleus of the trapezoid body, is particularly important for mediating inhibitory signals to the contralateral superior olivary complex, which plays a key role in interaural differencing. The superior olivary complex thus receives inhibitory output from the contralateral cochlear nucleus via the medial nucleus of the trapezoid body and excitatory signalling from the ipsilateral ventral cochlear nucleus $(19,20)$.

Impairment of any of these pathways in brainstem lesions involving the pons, as was the case in our patient, can be the cause of central hearing impairment (Figure 2-B) (15). Our patient presented with bilateral, asymmetric hypoacusis, which we considered secondary to his pontine haemorrhage. What was further particular in this case was the association of paradoxical hyperacusis. In previously reported cases, the most likely cause was the injury of the auditory fibres projecting to the inferior colliculi, above the superior olivary complex and the lateral lemniscus $(21,22)$. We assumed that the

\section{REFERENCES}

1. Kosty JA, Mejia-Munne J, Dossani R, Savardekar A, Guthikonda B. Jacques Jean Lhermitte and the syndrome of peduncular hallucinosis. Neurosurg Focus. 2019 Sep 1;47(3):E9.

2. Benke T. Peduncular hallucinosis: a syndrome of impaired reality monitoring. J Neurol. 2006 Dec;253(12):1561-71.

3. Penney L, Galarneau D. Peduncular hallucinosis: a case report. Ochsner J. 2014 Fall;14(3):450-2.

4. Caplan LR. Diagnosis: Clinical, imaging, and laboratory. Vertebrobasilar Ischemia and Hemorrhage: Clinical Findings, Diagnosis and Management of Posterior Circulation Disease. 2nd ed. Cambridge: Cambridge University Press; 2015:133-162.

5. Riley DS, Barber MS, Kienle GS, Aronson JK, et al. CARE guidelines for case reports: explanation and elaboration document. J Clin Epidemiol. 2017 Sep;89:218-235.

6. Galetta KM, Prasad S. Historical Trends in the Diagnosis of Peduncular Hallucinosis. J Neuroophthalmol. 2018 Dec;38(4):438-441.

7. Friedman JH, Chou KL. Chapter 3 - Mood, Emotion, and Thought. In: Textbook of Clinical Neurology, Third Edition, Philadelphia: W.B. Saunders; 2007:35-54.

8. Dogan VB, Dirican A, Koksal A, Baybas S. A case of peduncular hallucinosis presenting as a primary psychiatric disorder. Ann Indian Acad Neurol. 2013 Oct;16(4):684-6.

9. Gokce M, Adanali S. Peduncular hallucinosis due to brain metastases from cervix cancer: a case report. Acta Neuropsychiatr. 2003 Jun;15(3):105-7.

10. Hayashi Y, Yoshikura N, Kimura A, Inuzuka T. Peduncular hallucinations in brainstem encephalitis drawn by a patient. Neurology. 2012 Oct 9;79(15):1625.

11. Nicolai A, Lazzarino LG. Peduncular hallucinosis as the first manifestation of multiple sclerosis. Eur Neurol. 1995;35(4):241-2.

12. Miyazawa T, Fukui S, Otani N, Tsuzuki N, Katoh H, Ishihara S, Nawashiro H, Wada K, Shima K. Peduncular hallucinosis due to a pineal meningioma. Case report. J Neurosurg. 2001 Sep;95(3):500-2. haemorrhage also led to a dysfunction of the right trapezoid body and the right superior olivary complex, which represent the first center of bilateral auditory integration in the brainstem (23). In support of this hypothesis, the follow-up MRI of the patient showed subtle signs of hypertrophic secondary olivary degeneration. However, involvement of the ventral acoustic striae that decussate in the trapezoid body could equally explain the bilateral hearing loss (23). A phenomenon of hypersensitization secondary to the damage of auditory sensory fibres most likely occurred in addition to the loss of inhibitory output from the right medial nucleus of the trapezoid body, contributing to the paradoxical left hyperacusis $(22,24)$.

\section{CONCLUSIONS}

Peduncular hallucinosis is a rare syndrome usually caused by lesions in the upper brainstem. Associations of auditory hallucinations with hypoacusis and paradoxical hyperacusis, as was the case of our patient, has been rarely reported in literature. Further brain networking mapping studies are definitely needed to enrich our current understanding of such complex auditory impairments.

Conflict of interest: none declared Financial support: none declared

13. Celesia GG. Hearing disorders in brainstem lesions. Handb Clin Neurol. 2015;129:509-36.

14. Braun CM, Dumont M, Duval J, Hamel-Hébert I, Godbout L. Brain modules of hallucination: an analysis of multiple patients with brain lesions. J Psychiatry Neurosci. 2003 Nov;28(6):432-49.

15. Häusler R, Levine RA. Auditory dysfunction in stroke. Acta Otolaryngol. 2000 Sep;120(6):689-703.

16. Kim NY, Hsu J, Talmasov D, Joutsa J, Soussand L, Wu O, Rost NS, Morenas-Rodríguez E, Martí-Fàbregas J, Pascual-Leone A, Corlett PR, Fox MD. Lesions causing hallucinations localize to one common brain network. Mol Psychiatry. 2021 Apr;26(4):1299-1309.

17. Celesia GG. Hearing disorders in brainstem lesions. Handb Clin Neurol. 2015;129:509-36.

18. Cohen M, Luxon L, Rudge P. Auditory deficits and hearing loss associated with focal brainstem haemorrhage. Scand Audiol. 1996;25(2):133-41.

19. Brown MC, Santos-Sacchi J. Chapter 25 - Audition. In: Squire LR, Berg D, Bloom FE, du Lac S, Ghosh A, Spitzer NC, editors. Fundamental Neuroscience, Fourth Edition. San Diego: Academic Press, 2013:553-76.

20. Kulesza RJ Jr, Grothe B. Yes, there is a medial nucleus of the trapezoid body in humans. Front Neuroanat. 2015 Mar 31;9:35.

21. Noureldine MHA, Kikano R, Riachi N, Ahdab R. Hyperacoustic hypoacusis: A new pontine syndrome - Case report. Brain Inj. 2017;31(10):1396-1397.

22. Lee E, Sohn HY, Kwon M, Kim JS. Contralateral hyperacusis in unilateral pontine hemorrhage. Neurology. 2008 Jun 10;70(24 Pt 2):2413-5.

23. Caplan LR (ed.). Stroke syndromes. In: Caplan's Stroke: A Clinical Approach. 5th ed. Cambridge: Cambridge University Press; 2016:131143.

24. Lee $\mathrm{E}$, Sohn HY, Kwon M, Kim JS. Contralateral hyperacusis in unilateral pontine hemorrhage. Neurology. 2008 Jun 10;70(24 Pt 2):2413-5. 